\title{
An Approximation Method of Bézier Curve
}

\author{
Zhi wu ${ }^{1}$, Chu yi Song ${ }^{1} \&$ De xi Bao ${ }^{1}$ \\ ${ }^{1}$ College of Mathematics, Inner Mongolia University for Nationalities, Tongliao, China \\ Correspondence: College of Mathematics, Inner Mongolia University for Nationalities, Tongliao 028043, China. \\ E-mail: zhiwu0103@163.com
}

Received: October 16, 2017

Accepted: October 24, 2017

Online Published: October 31, 2017

doi:10.5539/cis.v10n4p67

URL: http://doi.org/10.5539/cis.v10n4p67

\begin{abstract}
It is proved that the linear space constructed by power base is a banach space under 2-norm by using approximation method. For the Bézier curve--the elements in banach space, the linear combination of the low-order $\mathrm{S}$ power base is used to approximate optimal the high-order Bernstein base function. The original Bézier curve is instituted by the linear combination of low-order S power base and the optimal approximation element of the original Bézier curve is obtained.
\end{abstract}

Keywords: S power base, distance functions, optimal approximation, banach space

\section{Introduction}

Approximating the high-order polynomial curves and curved surfaces with the low-order ones plays an important role in data compression, data transmission, and data exchange, etc., in geometric modeling tasks. Generally, it is also an important research topic in Computer Aided Design (CAD). In recent years, the research on Bézier curve approximation with degree reduction attracts lots of attention. Current algorithms for Bézier Curve degree reduction can be summarized into two categories. The first category is based on the base function conversion. For instance in reference (L Z. Lu, and G Z Wang 2008) propose to utilize the Chebyshev Polynomials for base function conversion. In reference (Sánchez-Reyes J 1997), Sánchez-Reyes uses the S power base to accurately represent the Bernstein base function. Farouki proposes the degree reduction algorithm based on the Legendre Polynomials in reference (Rida T Farould 2000). The second category can be summarized as the geometric approximation algorithms with the control vertexes. In this category, reference (Forrest A R 1972) is based on the interpolation algorithm; S M. Hu proposes an approximation algorithm with the degree reduction; Reference (Sunwoo H and Lee N 2004) is based on the generalized matrix reduction technique. In reference (Jiuping. Cheng 1997), Cheng constructs the novel control vertexes based on the convex combinations of the classic control vertexes. Based on the Sánchez-Reyes's work, we further propose a degree reduction method with the optimal approximation. This method is built on the Bernstein base function and the properties of $\mathrm{S}$ power base.

\section{The Theoretical Problem}

In the normed space, the goal of approximation theory research includes two aspects: approximating a function in certain function space with the functions from its subspace, and constructing the optimal approximation according to the given criteria.

Definition 2.1 Suppose $M$ is a fixed non-empty subspace in the normed space $X$, for any $x \in X$, the distance between $x$ and $M$ is defined as:

$$
\rho==d(x, M)=\inf _{y \in M}\|x-y\|
$$

Suppose the $y_{0} \in M$ satisfies

$$
\left\|x-y_{0}\right\|=\rho
$$

then $y_{0}$ is called as the optimal approximation of $x$ in $M$. 
Proposition 2.2 Suppose $K$ is a number field, the set $\left\{0, \pm t^{i}\right\}_{i=0}^{n}(t \in[0,1])$ is a linear space in the $K$

It is easy to prove that the Proposition 2.2 is true.

Definition 2.3 Ssuppose $X=\left\{0, \pm t^{i}\right\}_{i=0}^{n}(t \in[0,1])$ is the linear space in the number filed $K$, we define the function

$$
\begin{gathered}
\|\bullet\|: X \rightarrow R^{1} \text { in } X \text {, where } \\
\|u\|=\left(\int_{[0,1]}|u(t)|^{2} d t\right)^{1 / 2} \cdot
\end{gathered}
$$

Proposition $2.4(X,\|\bullet\|)$ is the linear normed space in the number field $K$.

Therefore, the linea space $X$ is the banach space space.

Theorem 2.5 Suppose $M$ is a finite dimensional subspace in the normed space $X$, then for each $x \in X$, there exists an optimal approximation of $x$ in $M$.

Theorem 2.6 Suppose $X$ is a strictly convex normed space, the subspace $M \subset X$, then the maximum number of the optimal approximation of any $x \in X$ is 1 .

\section{Bézier Curve Approximation with Degree Reduction}

How to get a low-order curve which satisfied the condition to approximate the given Bézier curve?

Definition 3.1 (Sánchez-Reyes J 1997) given an integer $p \geq 1$, we define the following base function as the P-order S Power base function:

$$
\left.\left\{s^{k}(1-t), s^{k} t, s^{p}\right\}_{k=0}^{p-1} \Psi^{k} s^{k}(1-t), s^{k} t\right\}_{k=0}^{p-1}, s=(1-t) t, t \in[0,1]
$$

According to the above definition, if $n \geq 2 p$, then

$$
\left.\left\{s^{k}(1-t), s^{k} t, s^{p}\right\}_{k=0}^{p-1} \mathcal{Y} s^{k}(1-t), s^{k} t\right\}_{k=0}^{p-1} \subseteq\left\{0, \uplus^{i}\right\}_{i=0}^{n}, t \in[0,1]
$$

Form the proposition 2.2 and proposition 2.3, Bézier curve is a curve in normed space. And from theorem 2.5 and theorem 2.6, there is no more than one optimal approximation element for each element in the subspace of normed space.

Suppose $A_{n}(t)$ is a $n$-order Bézier curve, whose control vertexes are $p_{i}(i=0,1, \cdots, n)$

$$
A_{n}(t)=\sum_{i=0}^{n} p_{i} B_{i}^{n}(t) \quad t \in[0,1] .
$$

Where $B_{i}^{n}(t)=C_{i}^{n} t^{i}(1-t)^{n-i}, \tilde{A}_{n-m}(t)$ is the $m$-order optimal approximating function of the $n$-order Bézier curve $A_{n}(t)$. For each Bernstein base function, we reduce its degree and find its approximation in the following way:

Suppose $n$ is odd and the reduced degree is odd, 


$$
\begin{aligned}
& \min \left\|\frac{B_{i}^{n}(t)}{C_{i}^{n}}-\sum_{k=0}^{p-1}\left(a_{k}^{i} s^{k}(1-t)+b_{k}^{i} s^{k} t\right)-c_{p}^{i} s^{p}\right\|^{2} \\
& \text { s.t. } \quad 1-\sum_{k=0}^{p-1}\left(a_{k}^{i} s^{k}(1-t)+b_{k}^{i} s^{k} t\right)-c_{p}^{i} s^{p} \geq 0 \\
& \quad \sum_{k=0}^{p-1}\left(a_{k}^{i} s^{k}(1-t)+b_{k}^{i} s^{k} t\right)-c_{p}^{i} s^{p} \geq 0 \\
& i=0,1, \cdots, n
\end{aligned}
$$

Suppose $n$ is odd and the reduced degree is even,

$$
\begin{aligned}
& \min \left\|\frac{B_{i}^{n}(t)}{C_{i}^{n}}-\sum_{k=0}^{p-1}\left(a_{k}^{i} s^{k}(1-t)+b_{k}^{i} s^{k} t\right)\right\|^{2} \\
& \text { s.t. } \quad 1-\sum_{k=0}^{p-1}\left(a_{k}^{i} s^{k}(1-t)+b_{k}^{i} s^{k} t\right) \geq 0 \\
& \quad \sum_{k=0}^{p-1}\left(a_{k}^{i} s^{k}(1-t)+b_{k}^{i} s^{k} t\right) \geq 0 \\
& i=0,1, \cdots, n
\end{aligned}
$$

Suppose $n$ is even and the reduced degree is odd,

$$
\begin{aligned}
& \min \left\|\frac{B_{i}^{n}(t)}{C_{i}^{n}}-\sum_{k=0}^{p-1}\left(a_{k}^{i} s^{k}(1-t)+b_{k}^{i} s^{k} t\right)\right\|^{2} \\
& \text { s.t. } \quad 1-\sum_{k=0}^{p-1}\left(a_{k}^{i} s^{k}(1-t)+b_{k}^{i} s^{k} t\right) \geq 0 \\
& \quad \quad \sum_{k=0}^{p-1}\left(a_{k}^{i} s^{k}(1-t)+b_{k}^{i} s^{k} t\right) \geq 0 \\
& i=0,1, \cdots, n
\end{aligned}
$$

Suppose $n$ is even and the reduced degree is even,

$$
\begin{aligned}
& \min \left\|\frac{B_{i}^{n}(t)}{C_{i}^{n}}-\sum_{k=0}^{p-1}\left(a_{k}^{i} s^{k}(1-t)+b_{k}^{i} s^{k} t\right)-c_{p}^{i} s^{p}\right\|^{2} \\
& \text { s.t. } \quad 1-\sum_{k=0}^{p-1}\left(a_{k}^{i} s^{k}(1-t)+b_{k}^{i} s^{k} t\right)-c_{p}^{i} s^{p} \geq 0 \\
& \quad \sum_{k=0}^{p-1}\left(a_{k}^{i} s^{k}(1-t)+b_{k}^{i} s^{k} t\right)-c_{p}^{i} s^{p} \geq 0 \\
& i=0,1, \cdots, n
\end{aligned}
$$

The two inequalities in the constraint condition restrict the best approximating function fall within the range of the Bernstein base function with no coefficient. Because the optimal approximating function of $B_{i}^{n}(1-t){ }^{\text {is }}$ $\sum_{k=0}^{p-1}\left(a_{k}^{i} s^{k} t+b_{k}^{i} s^{k}(1-t)\right)$, and the optimal approximating function of $B_{i}^{n-i}(t)$ is 


$$
\sum_{k=0}^{p-1}\left(a_{k}^{n-i} s^{k}(1-t)+b_{k}^{n-i} s^{k} t\right)
$$

We can infer that

$$
\sum_{k=0}^{p-1}\left(a_{k}^{i} s^{k} t+b_{k}^{i} s^{k}(1-t)\right)=\sum_{k=0}^{p-1}\left(a_{k}^{n-i} s^{k}(1-t)+b_{k}^{n-i} s^{k} t\right) .
$$

According to the coefficient comparison, we get

$a_{k}^{i}=b_{k}^{n-i}, b_{k}^{i}=a_{k}^{n-i}$. (1) We have to satisfy the equality between the value of the Bernstein base function with no coefficient and the value of the optimal approximating function, on the vertexes in the domain of definition:

$$
\left\{\begin{array}{l}
\frac{B_{0}^{n}(0)}{C_{0}^{n}}=\frac{B_{n}^{n}(1)}{C_{n}^{n}}=a_{0}^{0}=b_{0}^{n}=1, \frac{B_{0}^{n}(1)}{C_{0}^{n}}=\frac{B_{n}^{n}(0)}{C_{n}^{n}}=b_{0}^{0}=a_{0}^{n}=0 \\
\frac{B_{i}^{n}(0)}{C_{i}^{n}}=\frac{B_{n-i}^{n}(1)}{C_{n-i}^{n}}=a_{0}^{i}=b_{0}^{n-i}=0, \frac{B_{i}^{n}(1)}{C_{i}^{n}}=\frac{B_{n-i}^{n}(0)}{C_{n-i}^{n}}=b_{0}^{i}=a_{0}^{n-i}=0 \\
1 \leq i \leq n-1
\end{array} .\right.
$$

Where $a_{k}^{i}, b_{k}^{i}$ and $a_{k}^{n-i}, b_{k}^{n-i}$ denote the coefficients of the optimal approximating functions of $\frac{B_{i}^{i}(t)}{C_{i}^{n}}, \frac{B_{i}^{n-i}(t)}{C_{i}^{n-i}}$, respectively. According to (1) and (2), we can get $n+1$ optimal approximating functions by computing the coefficients, i.e.,

$$
\left\{\begin{array}{l}
a_{k}^{i}, b_{k}^{i} \\
a_{k}^{i}, b_{k}^{i}, c_{p}^{i} \\
0 \leq i \leq\lfloor n / 2\rfloor, 1 \leq k \leq p .
\end{array}\right.
$$

\section{Approximation Error Estimation and Numerical Examples}

Suppose, $n>2 p-1$ or $n>2 p$ the error between the $n$-order Bézier curve and the curve after degree reduction

can be computed as

$$
e(t)=\sum_{i=0}^{n} p_{i}\left|B_{i}^{n}(t)-C_{i}^{n} \sum_{k=0}^{p-1}\left(a_{k}^{i} s^{k}(1-t)+b_{k}^{i} s^{k} t\right)\right| \text { or } e(t)=\sum_{i=0}^{n} p_{i}\left|B_{i}^{n}(t)-C_{i}^{n} \sum_{k=0}^{p-1}\left(a_{k}^{i} s^{k}(1-t)+b_{k}^{i} s^{k} t\right)-C_{i}^{n} c_{p}^{i} s^{p}\right| .
$$

Suppose $A_{9}(t)$ is a 9-order Bézier curve whose control vertexes is $p_{i}(i=0,1, \cdots, 9)$, and 5-order (Figure1.), 7-order (Figure2.) optimal approximations are illustrated in the following figures. 


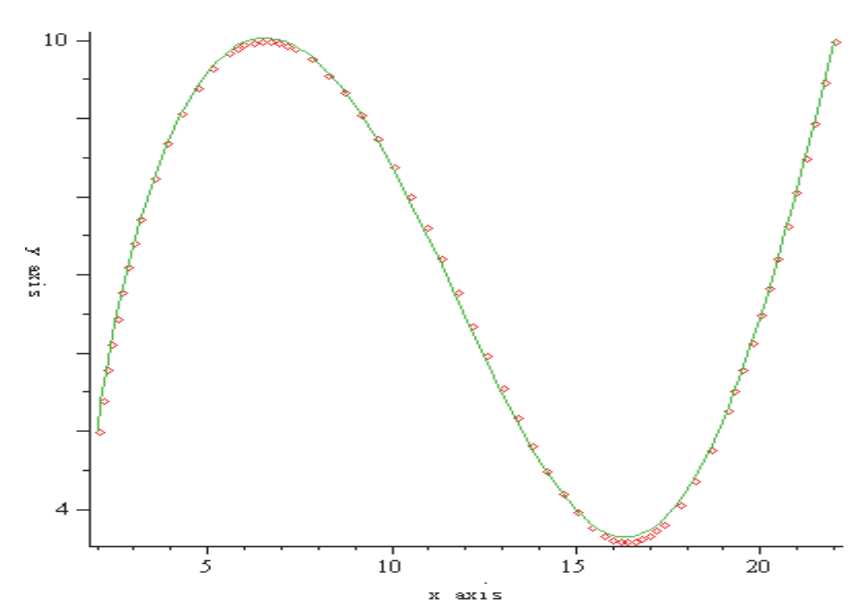

Figure 1.5-order optimal approximations curve

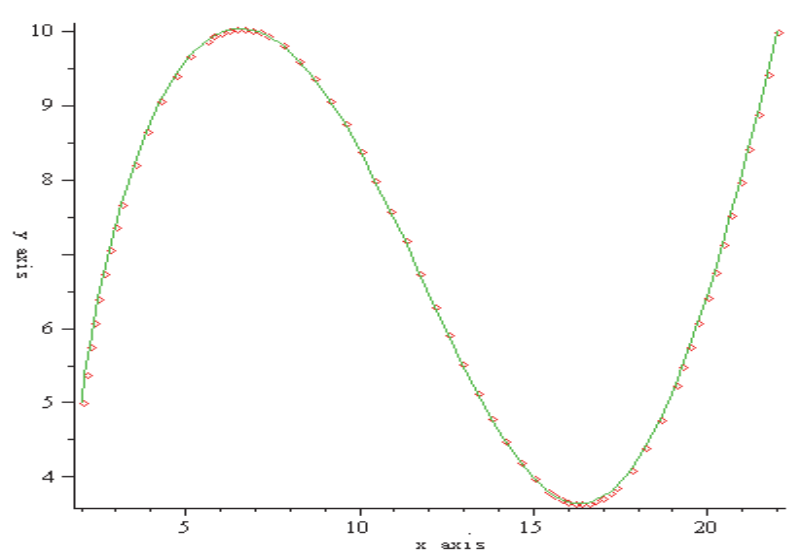

Figure 2. 7-order optimal approximations curve

In the two figures, 9-order Bézier curve is denoted by solid line; 5-order optimal approximations curve and 7-order optimal approximations curve are denoted by dashed line. The control vertexes are

$$
[2,5],[3,9],[6,12],[8,12],[11,9],[13,4],[15,2],[17,2],[19,4],[2,10]
$$

Respectively.The 9-order Bézier curve function $A_{9}(t)$ and the optimal approximating curve functions $\tilde{A}_{5}(t)$ and $\tilde{A}_{7}(t)$ are illustrated in the followings.

$$
\begin{aligned}
& A_{9}(t)=\left[\begin{array}{l}
x(t) \\
y(t)
\end{array}\right]^{T}=\left[\begin{array}{l}
2(1-t)^{9}+27 t(1-t)^{8}+216 t^{2}(1-t)^{7}+672 t^{3}(1-t)^{6}+1386 t^{4}(1-t)^{5}+1638 t^{5}(1-t)^{4}+1260 t^{6}(1-t)^{3}+612 t^{7}(1-t)^{2}+171 t^{8}(1-t)+22 t^{9} \\
5(1-t)^{9}+81 t(1-t)^{8}+432 t^{2}(1-t)^{7}+1008 t^{3}(1-t)^{6}+1134 t^{4}(1-t)^{5}+504 t^{5}(1-t)^{4}+168 t^{6}(1-t)^{3}+72 t^{7}(1-t)^{2}+36 t^{8}(1-t)+10 t^{9}
\end{array}\right] \\
& \tilde{A}_{7}(t)=\left[\begin{array}{l}
x(t) \\
y(t)
\end{array}\right]^{T}=\left[\begin{array}{l}
2-\frac{1577}{34} t^{3}(1-t)^{4}+20 t-\frac{4677}{442} t(1-t)^{2}+\frac{10498}{221} t^{2}(1-t)^{3}-\frac{361}{34} t^{4}(1-t)^{3}+\frac{6026}{221} t^{3}(1-t)^{2}-\frac{2973}{442} t^{2}(1-t) \\
5-\frac{1001}{34} t^{3}(1-t)^{4}+5 t+\frac{11601}{374} t(1-t)^{2}+\frac{16384}{221} t^{2}(1-t)^{3}-\frac{5221}{34} t^{4}(1-t)^{3}+\frac{4424}{221} t^{3}(1-t)^{2}-\frac{239247}{4862} t^{2}(1-t)
\end{array}\right]^{T} \\
& \tilde{A}_{5}(t)=\left[\begin{array}{l}
x(t) \\
y(t)
\end{array}\right]^{T}=\left[\begin{array}{l}
2+20 t+\frac{3204}{143} t^{3}(1-t)^{2}+\frac{348}{11} t^{2}(1-t)^{3}-\frac{892}{143} t^{2}(1-t)-\frac{1336}{143} t(1-t)^{2} \\
5+5 t-\frac{4626}{143} t^{3}(1-t)^{2}+\frac{8574}{143} t^{2}(1-t)^{3}-\frac{6454}{143} t^{2}(1-t)+\frac{4646}{143} t(1-t)^{2}
\end{array}\right]^{T}
\end{aligned}
$$

From the figure, it is intuitive to see that our method produces satisfying curve approximation. 
Table 1. Error estimation

\begin{tabular}{|c|c|c|c|c|c|c|c|}
\hline $\begin{array}{l}\text { Curves and } \\
\text { Error } \\
\text { Estimation }\end{array}$ & $\mathrm{x}$ & 3 & 7 & 11 & 13 & 16.5 & 19 \\
\hline $\begin{array}{l}\text { 9-order Bézier } \\
\text { curve }\end{array}$ & $y$ & $\begin{array}{l}7.44946352 \\
7 \\
\end{array}$ & $\begin{array}{l}10.0089303 \\
6\end{array}$ & $\begin{array}{l}7.46491162 \\
9\end{array}$ & $\begin{array}{l}.48441430 \\
1 \\
\end{array}$ & $\begin{array}{l}3.65111650 \\
7\end{array}$ & $\begin{array}{l}5.17081556 \\
5\end{array}$ \\
\hline $\begin{array}{l}5 \text {-order optimal } \\
\text { approximations } \\
\text { curve }\end{array}$ & $y_{1}$ & $\begin{array}{l}7.45059689 \\
3\end{array}$ & $\begin{array}{l}9.96598573 \\
0\end{array}$ & $\begin{array}{l}7.50847341 \\
2\end{array}$ & $\begin{array}{l}5.53232209 \\
0\end{array}$ & $\begin{array}{l}3.59573110 \\
7\end{array}$ & $\begin{array}{l}5.18348135 \\
9\end{array}$ \\
\hline $\begin{array}{l}\text { 7-order optimal } \\
\text { approximations } \\
\text { curve }\end{array}$ & $y_{2}$ & $\begin{array}{l}7.44640955 \\
2\end{array}$ & $\begin{array}{l}10.0093470 \\
6\end{array}$ & $\begin{array}{l}7.46266645 \\
7\end{array}$ & $\begin{array}{l}5.48451677 \\
9\end{array}$ & $\begin{array}{l}3.64999189 \\
4\end{array}$ & $\begin{array}{l}5.17487785 \\
9\end{array}$ \\
\hline \multirow{2}{*}{$\begin{array}{l}\text { Error } \\
\text { estimation }\end{array}$} & $\left|y-y_{1}\right|$ & $\begin{array}{l}0.00113336 \\
6\end{array}$ & $\begin{array}{l}0.04294463 \\
0\end{array}$ & $\begin{array}{l}0.04356178 \\
3\end{array}$ & $\begin{array}{l}0.04790778 \\
9\end{array}$ & $\begin{array}{l}0.05538540 \\
0\end{array}$ & $\begin{array}{l}0.01266579 \\
4\end{array}$ \\
\hline & $\left|y-y_{2}\right|$ & $\begin{array}{l}0.00305397 \\
5\end{array}$ & 0.00041670 & $\begin{array}{l}0.00224517 \\
2\end{array}$ & $\begin{array}{l}0.00010247 \\
8\end{array}$ & $\begin{array}{l}0.00112461 \\
3\end{array}$ & $\begin{array}{l}0.00406229 \\
4\end{array}$ \\
\hline
\end{tabular}

\section{Conclution}

This paper proposes an intuitive and effective method for n-order Bézier curve approximation. Experiments manifest that our method could get the optimal low-order approximations for the Bézier curves. In the further work, we will further take the non-negativity of the optimal approximating function into consideration, improve the precision of the low-order approximation, and improve the function approximation scale and the constraint condition.

\section{Acknowledgments}

This work is supported by the National Science Fund of China (NSFC11561052) and the Inner Mongolia Natural Science Foundation of China (NMDGP1415).

\section{References}

Cheng, J. P. (1997). Degree reduction of Bézier curves and algorithm of error calculation. Journal of An Hui Industrial College, 16, 55-58.

Forrest, A. R. (1972). Interactive interpolation and approximation by Bézier polynomials. The Computer Journal, pp.71-79 .

Hu, S. M., Sun, J. G., Jin, T. G., \& Wang, G. Z. (1998). Approximate degree reduction of Bézier curves. Tsinghua Science and Technology, 3, 997-1000.

Lu, L. Z., \& Wang, G. Z. (2008). Application of Chebyshev II-Bernstein basis transformations to degree reduction of Bézier curves. Journal of Computational and Applied Mathematics, 221, 52-65.

Rida, T. F. (2000). Legendre-bernstein basis transformations. Journal of Computational and Applied Mathematics, 119, 145-160.

Sánchez-Reyes, J. (1997). The symmetric analogue of the polynomial power basis. ACM Transactions on Graphics, 16, 319-357.

Sunwoo, H., \& Lee, N. (2004). A unified matrix representation for degree reduction of Bézier curves. Computer Aided Geometric Design, 21, 151-164.

\section{Copyrights}

Copyright for this article is retained by the author(s), with first publication rights granted to the journal.

This is an open-access article distributed under the terms and conditions of the Creative Commons Attribution license (http://creativecommons.org/licenses/by/4.0/). 